\title{
A Robot Centric Perspective on the HRI Paradigm
}

\author{
Nathan Kirchner, Alen Alempijevic \\ Centre for Autonomous Systems, Faculty of Engineering and Information Tech- \\ nology, University of Technology, Sydney
}

The industrial revolution undoubtedly defined the role of machines in our society, and it directly shaped the paradigm for human machine interaction - a paradigm which was inherited by the field of Human Robot Interaction (HRI) as the machines became robots. This paper argues that, for a foreseeable set of interactions, reshaping this paradigm would result in more effective and more often successful interactions. This paper presents our Robot Centric paradigm for HRI. Evidence in the form of summaries of relevant literature and our past efforts in developing social-robotics enabling technology is presented to support our paradigm. A definition and a set of recommendations for designing the key enabling component, sociocontextual cues, of our paradigm are presented. Finally, empirical evidence generated through a number of experiments and field studies $(N=456$ and $N$ $=320$ ) demonstrates our paradigm is both feasibly incorporated into HRI and moreover, yields significant contributions to the successfulness of a set of HRIs.

Keywords: Robot Centric Human-Robot Interaction, Participant roles, Interaction roles, Non-verbal cues, Sociocontextual cues

\section{Introduction}

There is a significant desire within society to see useful robots ubiquitous in our day-to-day lives. Whilst this desire is somewhat fuelled by Hollywood's depictions of robotics in the movies, it has a underpinning of real need. For instance, the global ageing population phenomena poses significant challenges. The Australian Treasury 2010 Intergenerational Report (Australia \& Swan, 2010) suggests that health spending on those aged over 65 is likely to increase sevenfold by 2050 as the number of working-age-persons per older-person is expected to fall from the present ratio of 5:1 to 2.7:1. Globally, we are moving to a situation of increased strain on the aged services and we are searching to facilitate ageing-in-place; robotics is widely acknowledged as a potential enabling technology. This is just one example of a real need that is motivating society's desire to see robots ubiquitous.

The concept of intelligent machines assisting people is pervasive in many technological tools in use today. The effectiveness of the da-Vinci robot system that assists surgeons in complex procedures is now supported by level II evidence (Geller, Siddiqui, J.M.Wu, \& Visco, 2008). Robotic

\footnotetext{
Authors retain copyright and grant the Journal of Human-Robot Interaction right of first publication with the work simultaneously licensed under a Creative Commons Attribution License that allows others to share the work with an acknowledgement of the work's authorship and initial publication in this journal.
}

Journal of Human-Robot Interaction, Vol. 1, No. 2, 2012, Pages 135-157. DOI 10.5898/JHRI.1.2.Kirchner 
telepresence has proven itself through deployments in dangerous or unreachable environments, with applications ranging from nuclear material handling demonstrated at the Fukushima nuclear plant disaster in 2011 to planetary exploration with the Mars Rover. Telepresence for teleconsultation and telehomecare are the subject of numerous trials in Australia and overseas, (Quan, Niwa, Ishikawa, Kobayashi, \& Kuno, 2011 for instance). Force extenders that support workers in lifting heavy loads are commonplace in the manufacturing industry (Bock, Linner, \& Ikeda, 2012). While these examples concern devices that are expensive and have complex interfaces requiring significant training, recent advances in machine intelligence together with the availability of low-cost sensing and computing platforms have now made it possible to build affordable robots aiming at a typical consumer market that incorporate these capabilities.

This, along with the previously mentioned needs, has given strength to the emerging field of assistive social robots; robots that are able to co-exist collaboratively with humans, as assistants, as tools, and as companions. However, a number of challenges remain open in order to achieve an appropriate degree of societal integration and acceptance for robots in this specific application space. These machines must become intelligent agents capable of perceiving and understanding human behaviour and needs, communicating with humans in a human-appropriate manner, and responding safely, efficiently and proactively to directions whether they be explicit or implicit. Furthermore, they must imitate the rich behaviours typically displayed by humans when acting as social entities in order to elicit particular expected behaviours from the interacting humans and/or to lessen the risk of social rejection. As a prerequisite to this, the machine must be capable of the non-trivial challenge of exhibiting behaviours perceived to be congruent with that a human would issue in a similar context (Ishiguro, 2011). Alternatively the machine could issue simplified behaviours/cues if, whilst clearly not congruent with that of a human, they were interpreted with an equivalent meaning. If feasible, this approach would allow lesser-humanistic ${ }^{1}$, and thus more achievable (in terms of their required technical capabilities and physical form), to fulfil this requirement. Our research focus questions at the core of this are: How could the paradigm for Human Robot Interaction (HRI) in the aforementioned application/interaction space be shaped to enable such agency? Can a lesserhumanistic robot exhibit behaviours/cues that are reliably interpreted by human interaction partners as intended? What form would such behaviours/cues take?

The breakdown of this paper is as follows: Section 2 presents a literature review illustrating the current paradigm for HRI and highlighting areas of potential opportunity. Section 3 presents our evidenced perspective on the paradigm shape required to enable robots to move into society. Following this, Section 4, describes and details our work, guided by this perspective, on sensing and perception of human cues. Section 5, describes our work, guided again by this perspective, on designing cues for eliciting a particular human response, and details two large scale field studies in which we explore real-world embodiments of our paradigm for HRI. Findings from our explorations of our paradigm are discussed, conclusions are drawn and future work is proposed in Section 6.

\section{Related Literature}

Undoubtedly, the role of machines was largely defined during the industrial revolution and it was this promising role that lead to the emergence of the field of robotics. However, these origins have directly shaped the paradigm of a robot's role in a task to be perceived as that of the Task Completer (Groom, 2008). Figure 1 illustrates this paradigm which originally consisted simply of the human acting upon a machine, perhaps simply pressing 'the button', to complete a task. Over time two additional feedback mechanisms were introduced. The first sees the machine provide 'Cues' back

\footnotetext{
${ }^{1}$ The term humanistic references to the set of human traits/attributes/affects encapsulated in the perspective presented by the field of Humanistic Psychology (Colman, 2009).
} 


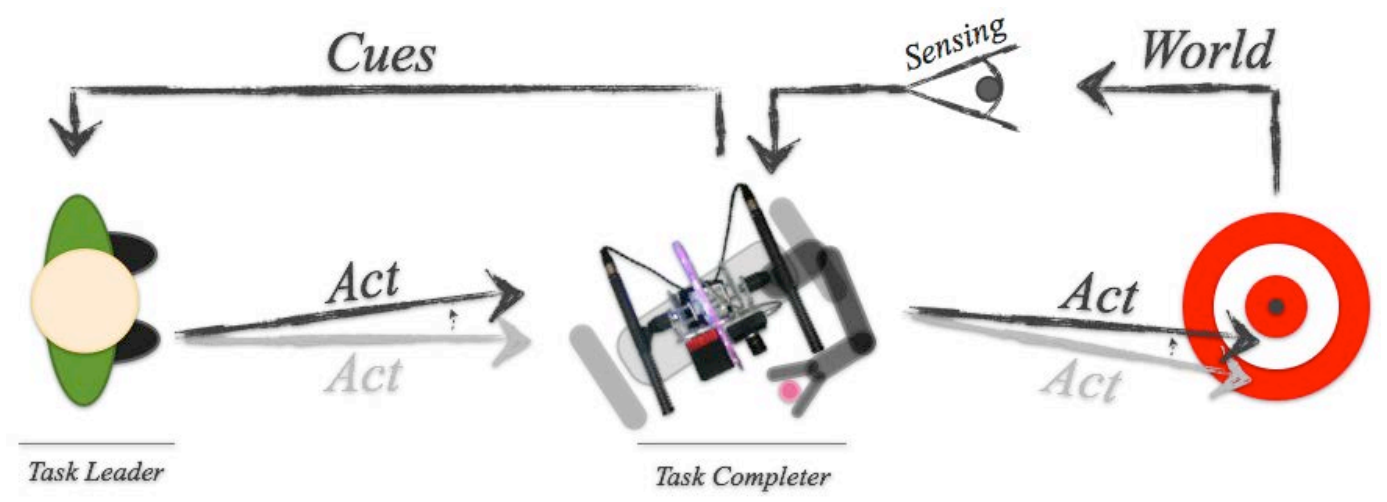

Figure 1. The traditional paradigm for human-machine/robot interaction

to the human; typically information such as task progress, performance, status, etc. is feedback to the human. The second sees the machine actively sense the 'World' in order to self-evaluate and self-correct; typical sensing information such as distance to target, rate of task completion, etc. are feedback to the machine's control/action strategy. Whilst the addition of these feedback pathways are well known to improve task performance and interaction, they have not altered the paradigm's fundamental shape.

Common approaches guided by this perspective (Rahimi \& Karwowski, 1990 and Robins et al., 2010 for example) see the human as central in the interaction paradigm; the human leads the interaction, communicates the task to be done and defines the intentionality of the situation. Conversely, the robot is a passive, albeit intelligent, participant at this level of the interaction and is the Task Completer; which is problematic for social robotics. Whilst this human-centric interaction paradigm is appropriate for task-defined operations such as Urban Search and Rescue (Burke \& Murphy, 2004), it is less appropriate in scenarios where robots and humans are peers, and/or the human/s are unaware of the robots task/intentions (demonstrated in Severinson-Eklundh, Green, \& Huttenrauch, 2003 and Kirchner, Alempijevic, \& Dissanayake, 2011 for example).

Consider the exemplar scenario shown in Figure 2. In this scenario the Task Leader instructs the lesser-humanistic robot to deliver an object to a particular member of a task-naive group, shown in Figure 2(a). This task-scenario, which is foreseeable in the context of social robotics, is problematic for the traditional HRI paradigm shown in Figure 1. Specifically, an individual who is naive to the task has unwittingly landed in the role of the Task Leader, shown in Figure 2(b). At best the task will be ill-defined in this individual's mind. As such, leading the interaction and/or interpreting cues from the robot is problematic, and in some cases the individual may simply ignore the robot and walk away (as was demonstrated prevalent by Satake et al., 2009, Dautenhahn, 2007 and Finke et al., 2005 for example). New requirements arise in such a scenario: instantiating an interaction, resolving ambiguity and shaping the participant roles.

Clearly then, in scenarios foreseeable in social robotics particular components of the task potentially should be Task Leader human defined, Task Naive human defined, robot defined or jointly defined. Thus, depending on the immediate task, the leadership role in the interaction may best be placed with the human, robot or jointly. Herein lies the limitation imposed by the traditional paradigm, there is a need for social robots to move from the role of the Task Completer and into the role of an Interaction Peer; an Interaction Peer will assume the role of Task Leader, Task Completer or Task Naive as appropriate during the interaction. The question thus becomes, How can the 


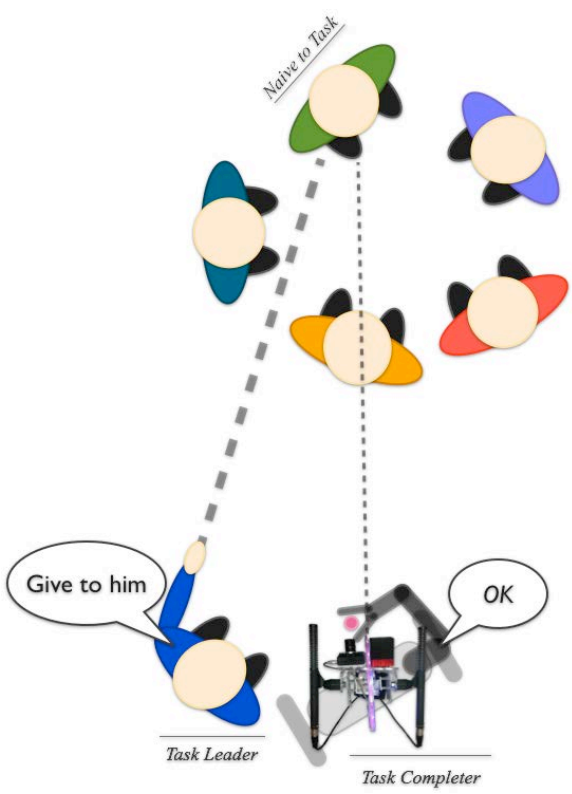

(a) The Task Leader instructs the lesser-humanistic robot to give an object to a particular member of a group. The group is not privy to the intended recipient.

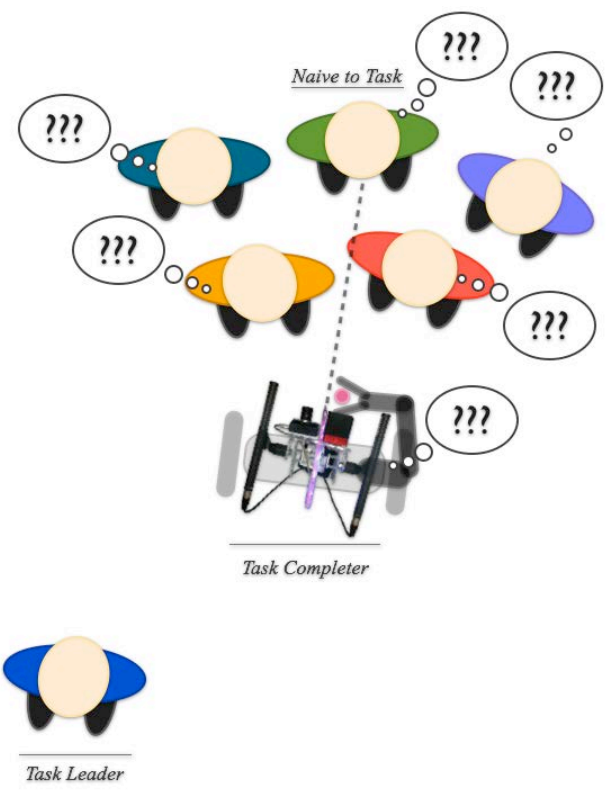

(b) Upon arrival, this task-naive group member has unwittingly become key to the interaction.

Figure 2. Exemplar Scenario

paradigm be reshaped so that both the human and robot roles are that of an Interaction Peer?

Fortunately a wealth of Human Human Interaction literature exists in the fields of Psychology, Behavioural Science and Robotics that can be built upon. For instance, it has been well demonstrated that a person's attention and intention is revealed from observable cues such as head/shoulder/body pose and that another person's attention and intention can be influenced directly by these acts/cues (Imamoglu, 1975, Mehrabian, 1971, Brown, 1965 and Kendon, 2004 for instance). A roboticsdomain specific example is Social Signal Processing (SSP) (Vinciarelli, Pantic, Bourlard, \& Pentland, 2008) which focuses on enabling the robot to ascertain a wealth of information from the interacting human through processing cues. However, enabling machines to issue socially interpretable cues designed to elicit particular behaviour is not the primary focus. Furthermore, it has been well demonstrated that a a person's current behaviour can predict their future behaviour and that a person's current behaviour can influence/elicit particular behaviour from another person (Guerin, 1999, Brown, 1965, Kendon, 1977 and Kendon, 1973 for instance). Also, it is well known that we (humans) actively utilise the aforementioned during our interactions to negotiate our roles and to establish ourselves as interaction peers (Watzlawick, Bavelas, \& Jackson, 1967). A robotics-domain specific example is Mixed Initiative Interaction (MII) (Hearst, 1999) which demonstrates the benefits of bi-directional communication during a task by the interaction partners in the case where one partner is a machine. However a prerequisite assumption for MII is that of the human having some sense of the 'goal', albeit in the presence of uncertainties, that can be mutually driven towards through grounding. In the case where the interacting human is truly task-naive this assumption is tested. However, whether it is possible to build upon the aforementioned and enable a robot to adopt 


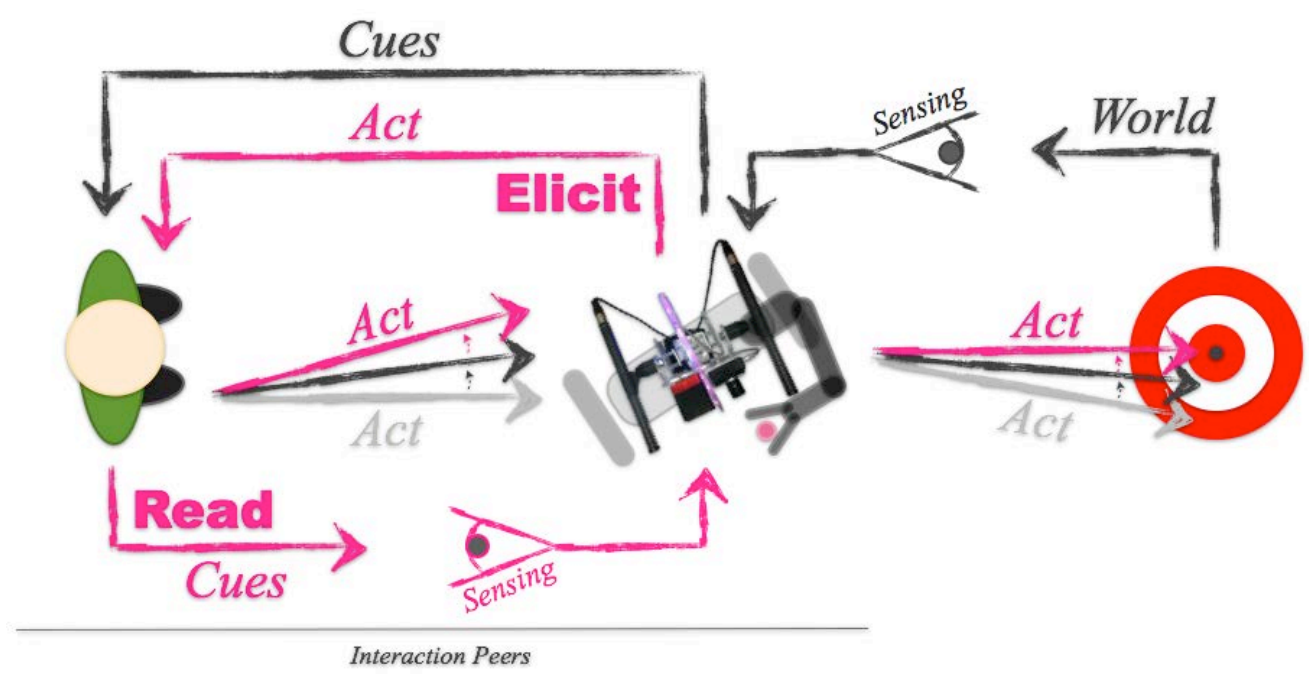

Figure 3. A paradigm for Robot Centric Human Robot Interaction

this strategy and assume the role of an Interaction Peer, thus reshaping the interaction paradigm, is a somewhat open question.

\section{Proposed Paradigm for Robot Centric HRI}

A Robot Centric HRI paradigm was devised in order to probe this question; Robot Centric in the sense that the robot has agency and is not limited to the role of Task Completer but is positioned as an Interaction Peer that can lead the interaction and resolve ambiguity in situations of naivety. Our paradigm for Robot Centric HRI, as presented in Figure 3, is built upon the foundations of the traditional paradigm but has had two additional feedback mechanisms/branches added in order to capture and exploit the aforementioned wealth of knowledge in the fields of Psychology and Behavioural Science. The branch labelled Read depicts the addition of the capacity for the robot to sense and subsequently perceive (read) the behavioural cues being displayed by the human. The second branch, labelled Elicit, indicates the addition of the ability of the robot to present behavioural acts designed to elicit particular behavioural responses from the interacting human; whereas, the traditional model's cues were primarily intended to provide information to the human upon which they may or may not choose to act. These branches and our exploration of them are detailed in the following sections. However, in order to provide insights into our interpretation of a lesserhumanistic robot, we will first present a brief note on our research platform.

Our mobile robot research platform is shown in Figure 4. The robot is based on a Segway RMP and is primarily instrumented with an XSens IMU, two Hokuyo UTM-30LX laser range finders, an RGB-D camera (the SwissRanger/Dragonfly combination has been replaced with a Microsoft Kinect), two Exact Dynamic iARM human-safe manipulators (an additional manipulator has been subsequently added). The robot stands approximately $1.5 \mathrm{~m}$ tall and weighs approximately $70 \mathrm{~kg}$. Through participation in RoboCup@ Home 2010 (Alempijevic et al., 2010) and 2011 (Alempijevic et al., 2011) and previous work (such as Caraian \& Kirchner, 2010, Richards, Paul, Webb, \& Kirchner, 2010 and O'Callaghan, Singh, Alempijevic, \& Ramos, 2011), we have developed a rich set of core capabilities: including capabilities in locomotion, navigation, path-planning, mobile manipulation, and sensing and perception. It is this platform and these capabilities that have facilitated our research 


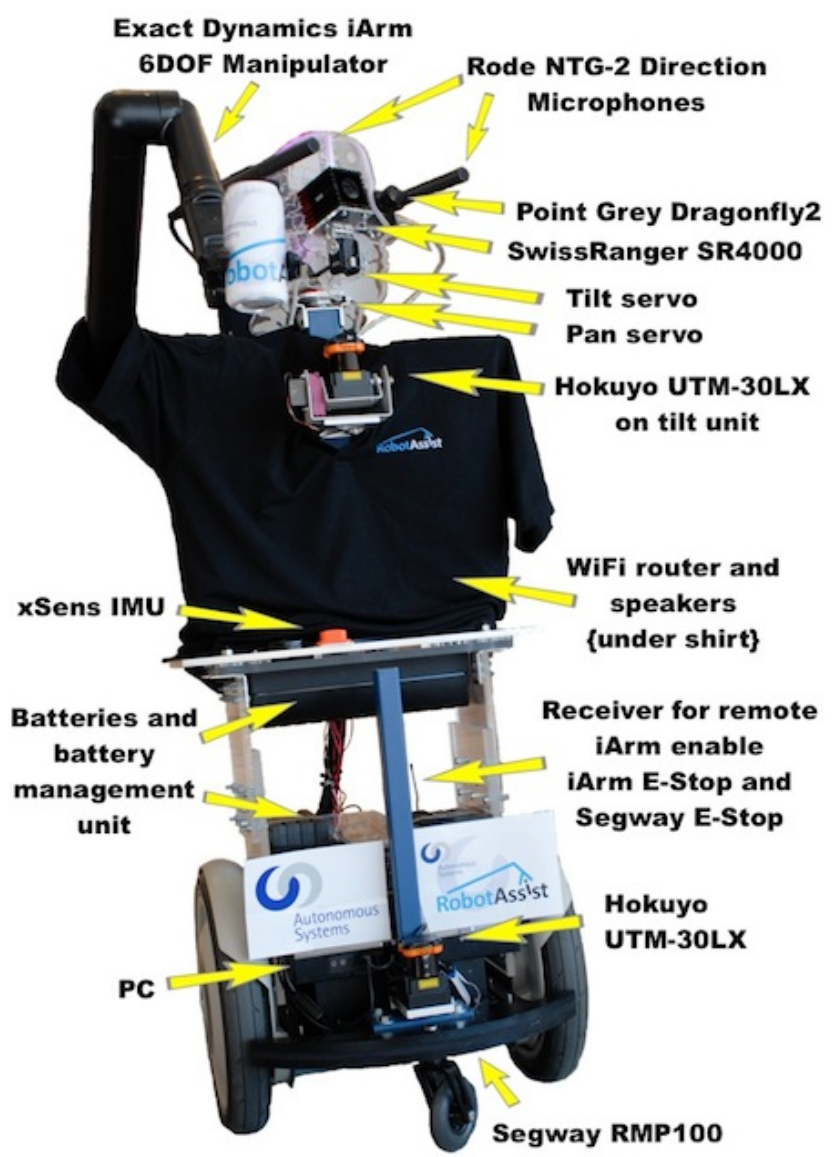

Figure 4. The RobotAssist Platform

probing these branches and our paradigm. Additional details of our platform are available from Kirchner \& et al., 2010 and www.robotassist.org

\section{The Read Branch: Sensing and Perception of Human Cues}

The Read branch, shown in Figure 3, is designed to enable the robot to sense the behavioural cues (such as the base cue of human presence, and deeper cues such as head direction, pose, gestures, etc.) being displayed by the interacting human/s. This sensing allows additional sources of richinformation to be obtained by the robot. This information is then interpreted through the robot's contextual understanding and through human behaviour-to-meaning mapping available from the fields of Psychology and Behavioural Science (a similar approach to that of Huang \& Mutlu, 2012). The resultant interaction-context knowledge is then exploited by the robot to derive an action plan. An example of our work in this branch and its contribution to HRI along these lines is given below.

The core cue that must first be robustly detected is that of human presence. A vision based system intuitively seems attractive and Open CV's Haar face detection algorithm is an example of such 


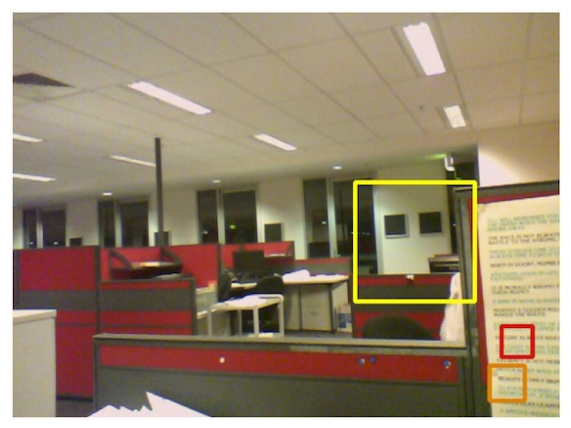

Figure 5. An example of OpenCV's Haar face detect's false positives

a system. However, vision based systems are inherently susceptible to a number of reasonable-toexpect occurrences such as variations in illumination conditions. Figure 5 shows such a case. In this figure, three 'faces', indicated by the superimposed rectangles, were detected by Open CV's Haar face detection algorithm. Whilst Haar face detection is arguably one of the leading approaches in terms of robustness and performance, clearly these particular detections are false positives. This is problematic as the occurrence of false positives reduces our ability to rely on the human detection and thus limits that which can built upon the output. For instance, a robot that attempted to interact with 'people' defined by a Haar detected face would have limited success in social robotics. Furthermore, Haar face detection is by no means the only technology that suffers similar false positives.

For these reasons we developed the 3D range-data based robust person detection technique presented in Hordern \& Kirchner, 2010. This technique uses projections of the 3D data on to a 2D horizontal plane and shape matching to enable segmentation. Fourier descriptors (FD) describing the segmentations are then created. Finally, a Support Vector Machine (SVM) trained with a range of differently shaped/sized people is then used to classify the observed as either Human or NonHuman. A database of 25 people was collected and used to test this approach. The results show that single-observation based, computationally efficient shape features can be used to robustly detect the presence of a person in the scene with an accuracy of $99.4 \%$. This technique is capable of detecting people in profile, facing away from the sensor, along with when facing the sensor. Furthermore, the technique is robust to a wider range of lighting conditions whilst maintaining comparable computational efficiency to OpenCV's Haar face detection.

This social robotics suitable approach to robust person detection has provided the front end required to alleviate the aforementioned false positive issues. Furthermore, with an input of a highconfidence point cloud segmentation labelled as being a person, additional valuable information becomes reliably available through exploiting anthropometrics. An example is shown in Figure 6. In this example, post robust person detection, Haar face detect on a depth image of the point cloud segmentation provides a bounding box for the face. The nearest point to the sensor within the box is the typically the nose, and the location of the nose relative to the bounding box indicates the head pose. Clearly this simplistic approach would suffer in a large search space, however it inherits robustness from the person detection approach and produces valuable information in this limited search space.

Another example in which we exploit the inherited robustness from the person detection front end to enable an otherwise potentially limited technique is in Kirchner, Alempijevic, \& Virgona, 2012. This method leverages the findings of an anthropometric survey of approximately 9000 soldiers by the US army which reported notable variations in the size and shape of peoples' head, neck 


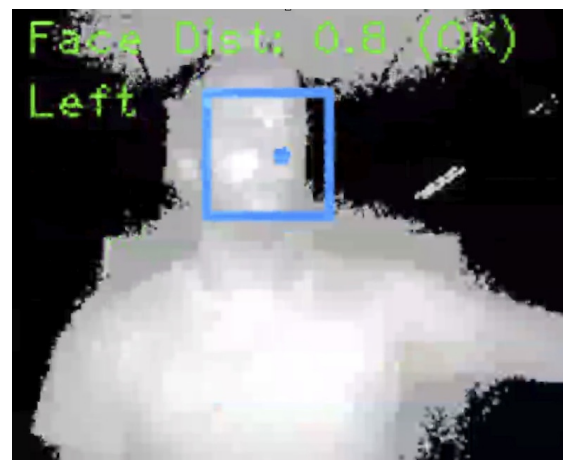

Figure 6. Haar face detect based head pose detection

and shoulders region (Gordon, Churchill, Clauser, Bradtmiller, \& McConville, 1989). Specifically, a scale and viewing angle robust feature vector constructed from analysing a 3D point cloud was designed to encapsulate this information; here within referred to as the Head-to-Shoulder Signature (HSS - shown in Figure 7). The HSS was then utilised as the basis for Support-Vector Machine (SVM) based robust in situ person recognition. Our results show that our feature vector constructed from a single observation is suitable for distinguishing between stationary and in-motion, both towards and directly away from the robot, individuals $(N=25)$ who are unaware that they are being scanned, with a mean accuracy of $78.15 \%$ and a peak accuracy of $100 \%$ for nine of the participants. The robustness of this approach is further apparent when considering that the results were collected over the five subsequent days from the training data collection day - i.e. post variations in the participants' general appearance.

In pursuit of additional information, robustness (through data fusion) and suitability for social robotics, a person detection approach based on a different sensing mode was devised. This approach to person detection (presented in Hilsenbeck \& Kirchner, 2011) is not 3D data reliant, but rather, is audio based. In this binaural approach the robot exploits the spectral structure (in the audio frequency domain) and characteristics specific to human-originating sound (speech or humming for instance) to robustly detect the presence and gender of human speakers. Our results show that our approach is capable of robustly and reliably detecting spectral patterns that conform to the spectral signature of speech, without reliance on a priori speaker knowledge. Furthermore, our results show that the speaker gender and bearing can also be reliably determined.

The previously mentioned examples of the Read branch sensing approaches are, however, only partially illustrative of the overall contribution of the branch. Another key contributor is the interaction-context knowledge, and subsequent action plan, derivable through the output of these technologies. For instance, take the exemplar scenario presented in Figure 8. In this scenario the robot has visually (RGB-D) and audibly (binaural) observed the scene; from the aforementioned a wealth of interaction-context knowledge is available. For instance, as shown in Table 1, the aforementioned sensing approaches together can reveal with high confidence: that two people spoke, the locations of these two people, who said what, and what was likely intended as a command to the robot; along with additional information such as the speakers' likely genders. Furthermore, with this interaction-context knowledge available, the formation of an informed and intelligent action plan becomes possible. For instance, in this case the action is $\langle$ Get Daniel a Coke $\rangle$. Clearly then, the Read branch has the ability to significantly contribute to the robot's interaction-context knowledge and action plan formation, and thus enables significant contribution to HRI. 


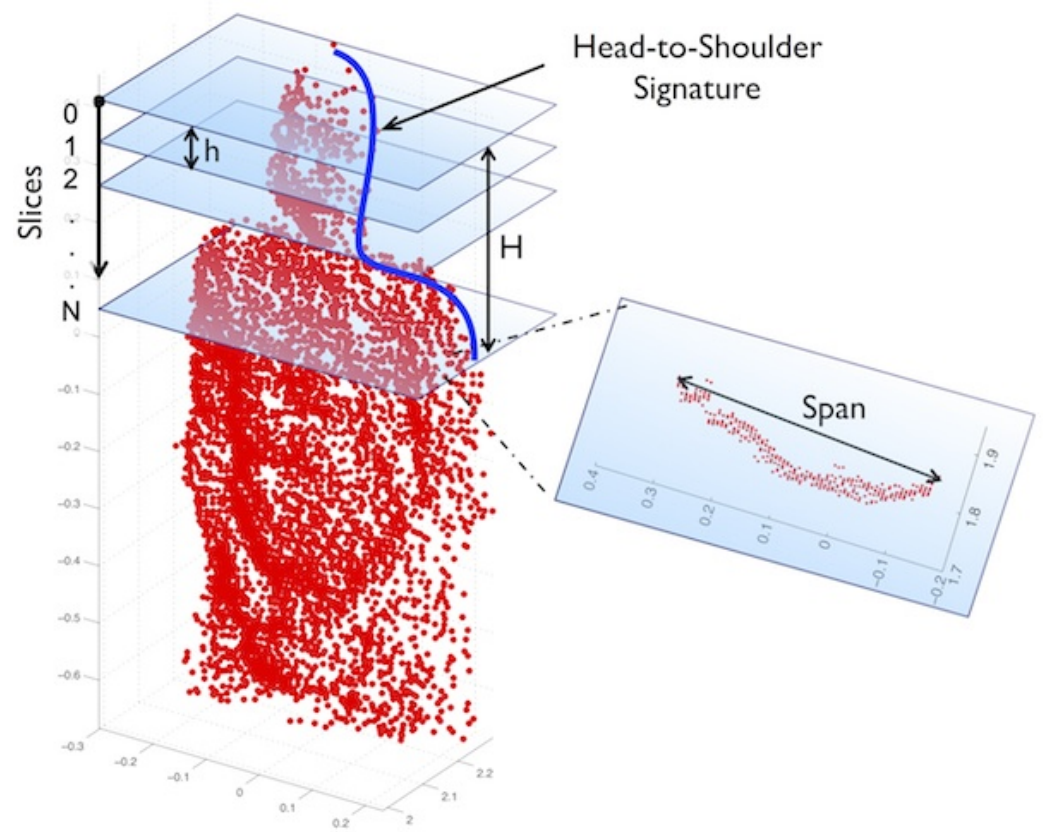

Figure 7. Segmented point cloud with the constructing elements of a HSS and a stylised HSS shown
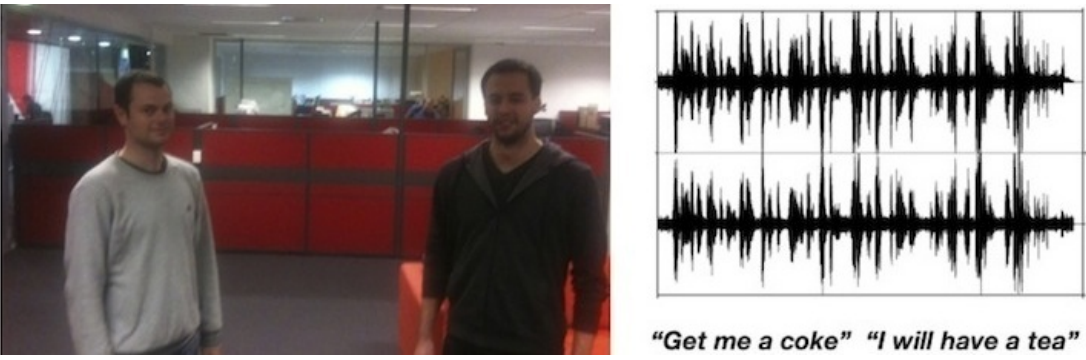

"Get me a coke" "I will have a tea"

Figure 8. Exemplar Scenario: The robot observes this scene visually and audibly 
Kirchner et al., A Robot Centric Perspective on HRI

Table 1: Interaction-context derivable from exemplar scenario

\begin{tabular}{|c|c|}
\hline Source & Output \\
\hline $\begin{array}{l}\text { 3D Person } \\
\text { Detection }\end{array}$ & $\begin{array}{l}\text { Two people detected with high-confidence. } \\
\text { Person } 1 \text { is a }\left\{\mathrm{x}_{1}, \mathrm{y}_{1}, \varnothing_{1}\right\} \text { and Person } 1 \text { is a }\left\{\mathrm{x}_{2}, \mathrm{y}_{2}, \varnothing_{1}\right\}\end{array}$ \\
\hline Head Pose & $\begin{array}{l}\text { Person } 2 \text { is looking at me (the robot). } \\
\text { Person } 1 \text { is looking at the bearing from themselves, to Person } 2 \text {. }\end{array}$ \\
\hline $\begin{array}{c}\text { Person } \\
\text { Recognition }\end{array}$ & Person 2 is Daniel. Person 1 is Gavin. \\
\hline Logic & Daniel is looking at me (the robot). Gavin is looking at Daniel. \\
\hline Interaction Model & Daniel is interacting with me (the robot). \\
\hline $\begin{array}{l}\text { Audio Person } \\
\text { Detection }\end{array}$ & $\begin{array}{l}\text { There were two sources of audio. Both are identified as human } \\
\text { originating with high-confidence increase the overall confidence } \\
\text { of the presence of two humans. Source } 1 \text { was at }-15^{\circ} \text { and is male, } \\
\text { Source } 2 \text { was at } 20^{\circ} \text { and is male; increases the overall confidence } \\
\text { of the location of the two humans The audio "I will have a tea" is } \\
\text { from Source } 1 \text {. The audio "Get me a coke" is from Source } 2 \text {. }\end{array}$ \\
\hline Logic & Source 1 is Gavin, $-15^{\circ}=\varnothing_{1}$. Source 2 is Daniel, $20^{\circ}=\varnothing_{2}$. \\
\hline Logic & Gavin said "I will have a tea". Daniel said "Get me a coke". \\
\hline Interaction Model & $\begin{array}{l}\text { Gavin was not looking at me (the robot) when he spoke "I will } \\
\text { have a tea". Gavin is not issuing me (the robot) a command. }\end{array}$ \\
\hline Interaction Model & $\begin{array}{l}\text { Daniel was looking at me (the robot) when he spoke "Get me a } \\
\text { coke". Daniel is issuing me (the robot) a command. }\end{array}$ \\
\hline
\end{tabular}

\section{The Elicit Branch: Issuing Cues to Elicit a Particular Response}

The Elicit branch, shown in Figure 3, depicts the addition of the ability for the robot to surreptitiously act upon the interacting human in such a way as to result in the interacting human modifying their behaviour; either to directly resolve ambiguity or to elicit specific human action or additional information. This branch provides the foundation of the robot's ability to probe the user for more information that can be added to the its contextual understanding. This branch could be used for explicit actions (such as the robot issuing verbal commands/requests to the humans), however it is well known that negative human responses are common when robots are perceived as issuing explicit commands or dictating task/interaction structure (Syrdal, Dautenhahn, Koay, \& Walters, 2009, Dautenhahn \& Saunders, 2011 and Mutlu, Bartneck, Ham, Evers, \& Kanda, 2011 for example). Clearly this is not appropriate in our case, as our aim is to bring robots to the role of a Interaction Peer along with the human, and not to move humans to the role of a Task Completer. Conversely, implicit actions, such as issuing non-verbal cues, have been demonstrated to be less susceptible to negative human response (Syrdal et al., 2009, Dautenhahn \& Saunders, 2011 and Mutlu et al., 2011 for example). Furthermore, Goren, Sarty, \& Wu, 1975 demonstrated that underlying rich humanistic cues are simplistic cue/s that when presented in isolation evoke a similar response. Specifically, Goren demonstrated that infants responded to simplistic primary shapes arranged in a face-like manner in much the same way as they did to actual faces. From this it seems potentially feasible that particular simplistic cue/s, which a lesser-humanistic robot is capable of exhibiting, may be interpreted similarly to specific rich cues. Finally, non-verbal cues are well known to result in rich, effective 
and expedient communication (Kendon, 2004, Mehrabian, 1971 and Gieselmann \& Waibel, 2005 for instance). Thus, our focus is one of implicit influence (eliciting particular behavioural responses) through this branch via the robot issuing non-verbal cues.

In order to devise non-verbal cues designed to communicate a specific message and that are suitable for a robot to enact, the fundamentals of non-verbal human cues were first examined. Fortunately a wealth of literature describing such cues exists in the field of Psychology and Behavioural Science (Mehrabian, 2007, Poyatos, 1997, Carton, Kessler, \& Pape, 1999 and Knapp \& Hall, 2009 are some key sources, and in particular Goren et al., 1975). The key points from these sources are: 1) Significant (both in terms of quantity and impact) communication can be conveyed non-verbally. 2) Complex cues are built through compounding simple cues. 3) Simple cues have well defined and known meanings through social norms. 4) Further meanings are ascribed to these cues based on the situation in which they are being interpreted (Context). 5) Fewer cues, or simple cues, require more context to interpret and are by nature more subtle. 6) A simplistic cue/s may exist beneath rich humanistic cues that when presented in isolation would be interpreted by a human similarly to the rich cue. From these sources and key points it seems that a simple cue which is heavily reliant on context will not only be enactable by a robot but will feasibly result in effective, expedient and surreptitious communication. These cues require social-interaction space and contextual-task space in order to be interpreted and, therefore for the sake of brevity will be referred to as sociocontextual cues in the remainder of this paper.

Two examples of Arm-based sociocontextual cues suitable for implementation on a robot are given in Figure 9 and two Gaze-based examples are given in Figure 10. All four are designed based on the that presented in (Mehrabian, 2007, Poyatos, 1997, Carton et al., 1999 and Knapp \& Hall, 2009). The first, shown in the top sequence in Figure 9, is an Offering cue. The key is the halfsecond, $100 \mathrm{~mm}$ extension of the end-effector (hand) while holding an object. The next, shown in bottom sequence in Figure 9, is a Protecting cue. The key is the half-second, 100mm retraction of the end-effector (hand) while holding an object. In both cues the arm can return to its initial position at $50 \mathrm{~mm} / \mathrm{s}$ without this being perceived as a cue. The remaining two cues are gaze based. The first, shown in the left sequence in Figure 10, is an Individualising cue. The key is the half-second dip and half-second raise of the eyes in the vertical plane while the head is directed at the target for individualisation. The other, shown in the right sequence in Figure 10, is an Attention Directing cue. The key is the fifth-of-a-second to-object and fifth-of-a-second from-object movement of the eyes. The movement during the Attention Directing cue need not be restricted to the vertical plane, however, the start and end point of the cue should be the same point.

Enabled by our platform's core capabilities, the technologies, approaches and methodologies developed during our exploration of the Read branch, and the aforementioned sociocontextual cues, several sizeable field studies have been designed and conducted. As the target application domain of our research is social robotics these studies have been designed to be real-world. Our general guiding prerequisites are: that our studies are out-of-the lab in a novel and non-controlled setting, that the participants are naive to robots, our platform and our study, that they are of reasonable scale in terms of participant numbers, and that the robot is as autonomous as possible so as to reduce the likelihood of our during-study actions/interventions/decisions inadvertently creating an effect in the study. The following subsections present two of our most recent field studies designed to explore our paradigm for Robot Centric HRI.

\subsection{Study 1: Individualising a particular member of a group}

Ensuring that a particular and unsuspecting member of a group is the recipient of a salient-item hand-over is a complicated interaction, however, it is one foreseeable in social robotics. Upon the robot's arrival it is likely that the group will reconfigure (Dusan \& Traum, 2007, Kendon, 1977 and 

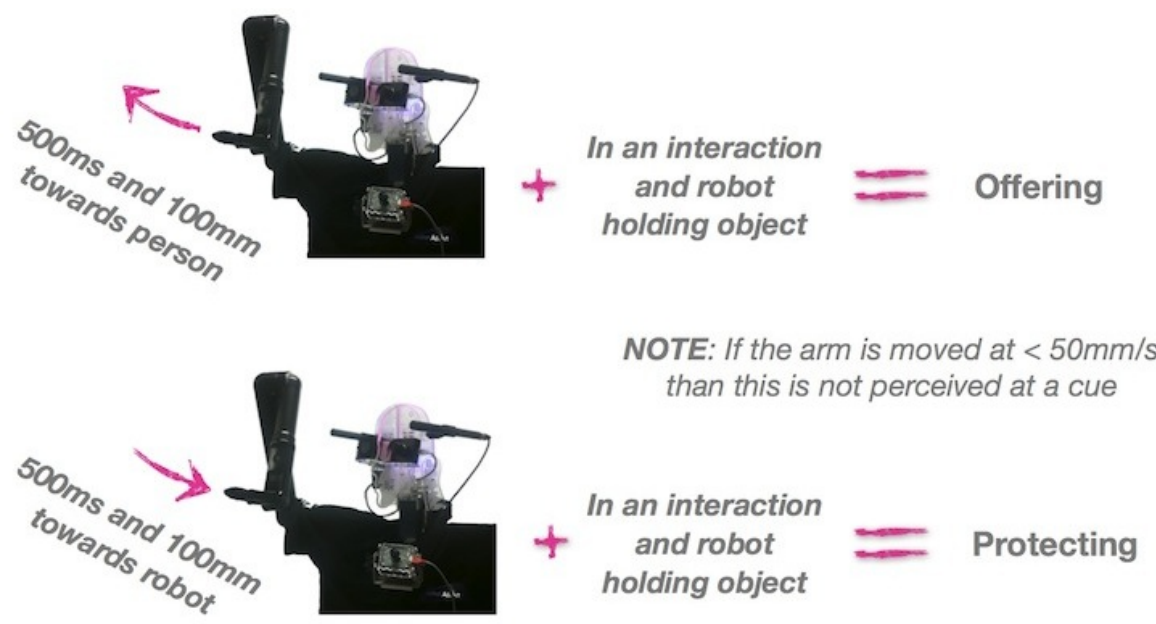

Figure 9. Sociocontextual Cues: Arm Based
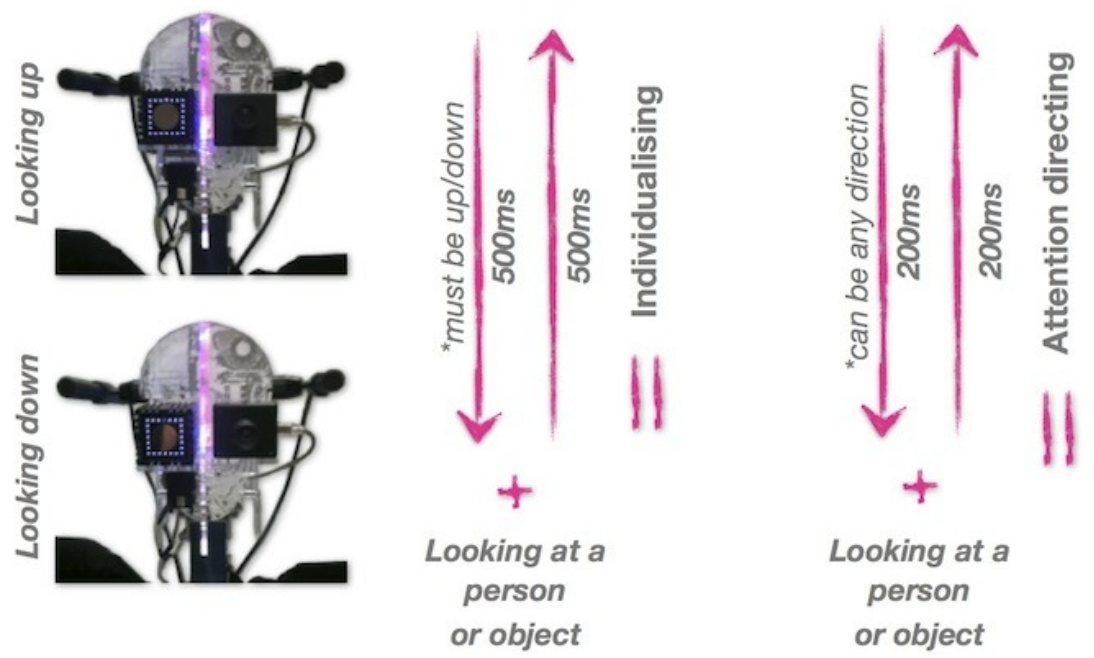

Figure 10. Sociocontextual Cues: Gaze Based 
Kendon, 1973) and, depending on the saliency of the object the robot is carrying, the group may actively compete for it (Guerin, 2003). In order to ensure the item is delivered to the correct person the robot must inform the intended recipient that the object is intended for him/her and, potentially more importantly, inform the remainder of the group that the object is not intended for them. This must be achieved by the robot in an effective, expedient, reliable and socially acceptable manner in order to advert any tendency within the group towards antinormative behaviour.

In the case of a salient object on 'offer' and a task-naive group, the lack of established social norms and ambiguity of the situation facilitates deindividuation within the group (Cowley, 2008 and Guerin, 2003). Furthermore, as robots are typically perceived as machines and not conscious beings this deindividuation will be exacerbated through an increased sense of anonymity (Postmes \& Spears, 1998) and the group is unlikely to feel subject to social influence or identification (Kiesler, Siegel, \& McGuire, 1984). This culmination of the lack of social influence and norms, and deindividuation, leads to antinormative behaviour (Diener, Fraser, Beaman, \& Kelem, 1976, Guerin, 2003 and Postmes \& Spears, 1998). In which case, the group-decision is likely to be polarised to either the group expediently dispossessing the robot of the salient object that it holds, or not acting in the scenario (Diener et al., 1976, Guerin, 2003 and Postmes \& Spears, 1998). However, as previously described, sociocontextual cues can individualise (reduce anonymity) and communicate intention (resolve ambiguity). Thus, it seems plausible for the robot to increase its likelihood of successful task completion by establishing appropriate participant roles. Which in turn seems plausible through the robot activating the Elicit branch by issuing particular sociocontextual cues. Testing these plausibilities was the focus of this study ${ }^{2}$.

The study was conducted during a University Open Day, shown in Figure 11(a). The participants ( $N=456$, the participant distribution is shown in Table 2) were visitors to the Open Day (primarily final stage high school students and their parents). They were attracted without experimenter intervention to participate in the study by the robot through its actions. The study was a $2 \times 2$ design; Gaze Based Individualising Sociocontextual Cue: Issued (Look) / Not Issued (No Look) x Participant Role: Interaction Partner (Selected) / By Stander (Onlooker). The arm based Offering sociocontextual cue was issued by the robot in all cases. The experimental procedure, illustrated in Figure 11(b), began with the robot autonomously making fairy floss (a.k.a. cotton candy). This novel spectacle enabled the robot to self-attract small groups (between 3 and 20 people). Once making the Fairy Floss was completed the robot looked up, scanned the crowd detecting the people and randomly selected an intended interaction partner from the pool of detected people. Finally, the cue case (Look or No Look) was randomly selected and issued whilst the arm based offering cue was executed, both cues were directed at the randomly selected interaction partner.

Whether the Fairy Floss was taken at all, the time elapsed prior to it being taken (if taken), whether it was taken by the selected participant or an onlooker, and a survey designed to probe the participants' perception of their entitlement to take the Fairy Floss were the measures used in this study. The driving hypotheses for the study was that: The robot's presentation of anonymityreducing sociocontextual cues will lead to perceived and enacted reduced equality of participation within the crowd. Our predictions were that: upon presenting such a cue, the robot will be more successful in confining the interaction to the selected participant. Specifically, there would be 1) a decreased likelihood that the object will be taken by an onlooker as opposed to the selected participant, 2) an increased likelihood that the object is taken, and 3) a perceived and thus reported greater sense of entitlement to take the item for the selected opposed to the onlookers.

Figure 12 presents a selection of the key results from this study. Evidence to support our hypotheses and predictions was found. As shown in Figure 12(a), it was found that the object was

\footnotetext{
${ }^{2}$ This study was initially presented in (Kirchner et al., 2011), this section presents a commentary on the role of this study in evaluating the paradigm presented here within, and presents previously unpublished additional findings/insights.
} 


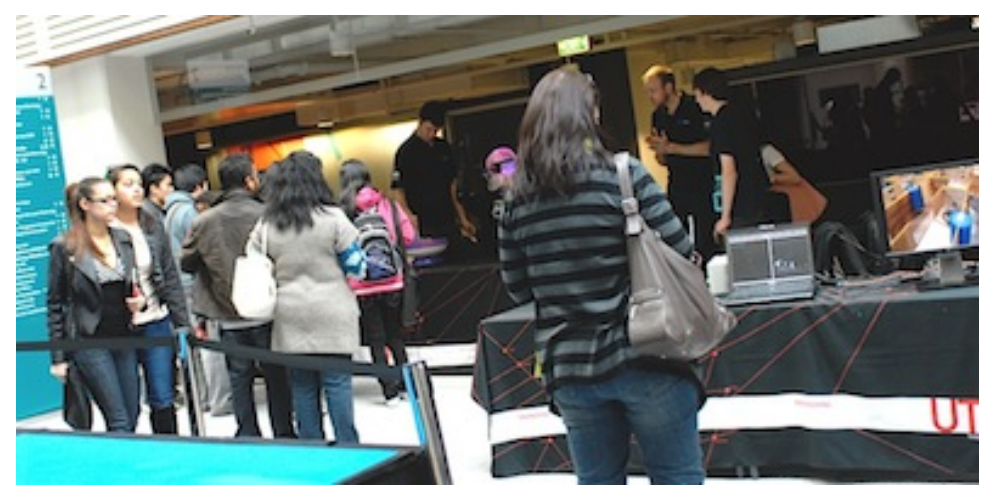

(a) A stand at a University Open Day

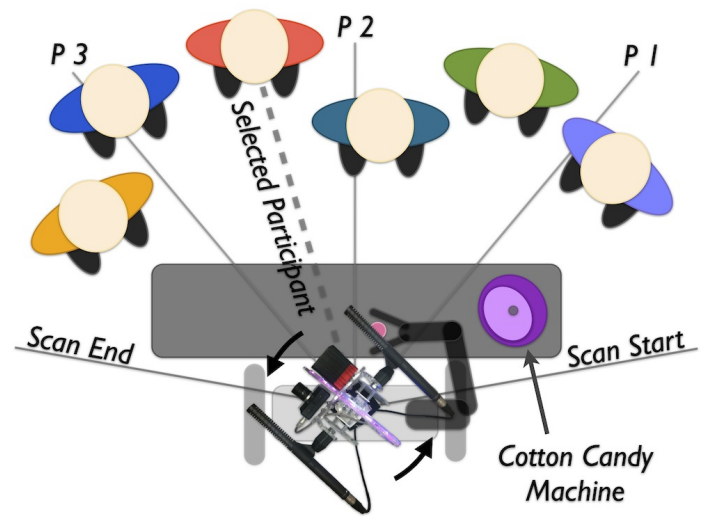

(b) An illustration of the experimental procedure

Figure 11. Exemplar Scenario

Table 2: Participant Distribution

\begin{tabular}{|c|c|c|c|c|}
\hline \multicolumn{2}{|c|}{ Case } & Onlooker & Selected & Total \\
\hline \multirow{2}{*}{ 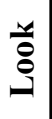 } & Male & 150 & 24 & 174 \\
\hline & Female & 85 & 16 & 101 \\
\hline \multirow{3}{*}{$\begin{array}{l}\frac{y}{8} \\
\stackrel{8}{\partial} \\
\stackrel{0}{Z}\end{array}$} & Male & 91 & 18 & 109 \\
\hline & Female & 66 & 6 & 72 \\
\hline & Total & 392 & 64 & 456 \\
\hline
\end{tabular}


Kirchner et al., A Robot Centric Perspective on HRI

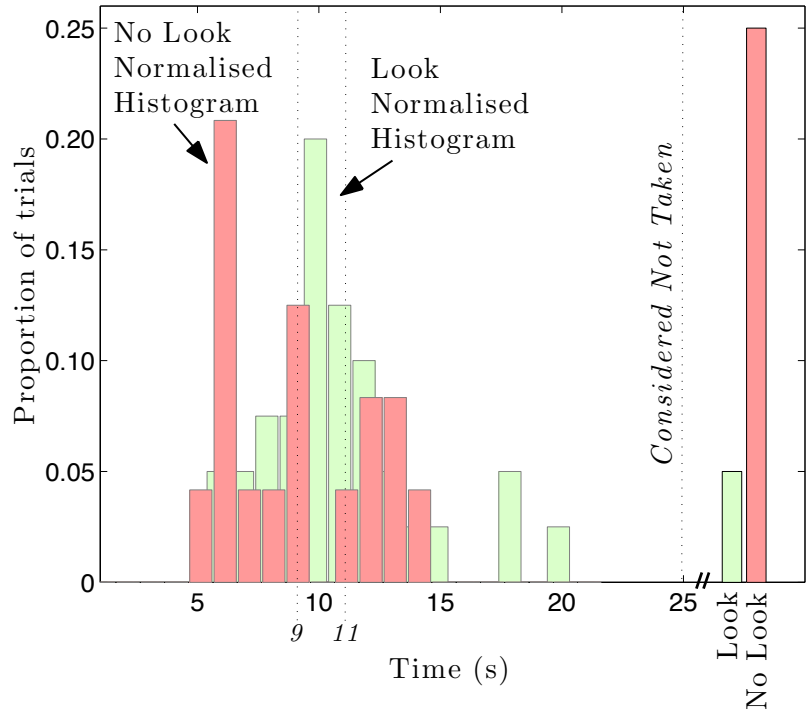

(a) Time elapsed after the gaze cue before the item was taken

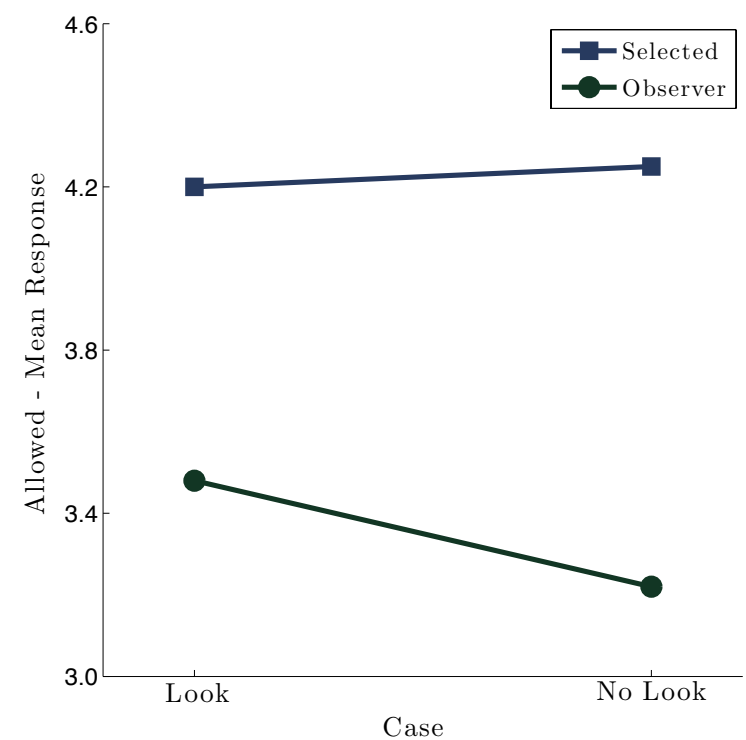

(b) Effect of Look Vs No Look on participants' percept on whether they are allowed to take the Item

Figure 12. Exemplar Scenario Results 
taken significantly more frequently by the selected rather than the onlooker when the Look cue was issued, $(z=3.73, p<0.01)$. No difference was found when the No Look cue was issued. Furthermore, it was found that the object was taken significantly more frequently when the Look cue was issued, $(p<0.01)$, and significant difference in the time-to-take the item between the two conditions was also found, $(t=2.42, p<0.02)$. Finally, of the 456 participants, 135 completed the optional survey. Figure 12(b) shows the effect of the presence (or absence) of the sociocontextual gaze cue on the participants' percept on whether they are allowed to take the item. As the responses to the survey items will be heavily influenced (by design) by the participant's role and experimental case, an overall test of internal reliability is inappropriate. Individual split measures of internal consistency see Cronbach's Alpha range from 0.61 to 0.80 (acceptable to good). Upon analysis a significant main effect for the participants' role (Selected Vs Observer) was found, $(F(1,129)=11.23, p<0.01)$. However, the main effect and simple main effects for the case were not found to be significant, nor was the interaction effect.

The results from this study demonstrate that a robot can assume the role of an Interaction Peer and defeat tendencies within the group towards antinormative behaviour through activating the Elicit branch of our Robot Centric paradigm for HRI with sociocontextual cues. Importantly, the results also show that the robot was significantly more able to complete its intended task (handover to a specific group member - $80 \%$ compared to $33.3 \%$ success $-z=3.73, p<0.01$ ) upon presenting the sociocontextual cue. Furthermore, our results also showed that the robot was able to achieve this surreptitiously, the participants' behaviour was effected as demonstrated by the success results, although the participants did not perceive a difference in entitlement to take the Fairy Floss between cases (non-significant main and simple main effects by case). From these results it is clear that the robot was able to activate the Elicit branch to its benefit without inducing a negative human response.

\subsection{Study 2: Instantiating an interaction with a particular passerby}

Successfully instantiating an interaction with a task-naive human is another example of a non-trivial, yet foreseeable, interaction requirement in social robotics. It has been found that interaction instantiation attempts based on increasing physical proximity and/or issuing greeting utterance often results in the human simply ignoring the robot (Satake et al., 2009, Dautenhahn, 2007 and Finke et al., 2005 for example). Whilst this is acceptable in a number of cases, it is not acceptable in the case where the robot must interact with a specific person. In order to ensure an interaction is instantiated with a desired person the robot must inform this person of its intentions in a compelling and directed (so as not to instantiate unintended interactions with onlookers) manner. This must be achieved by the robot in an effective, expedient, reliable and socially acceptable manner in order to advert any tendency towards antinormative behaviour.

Instantiating an interaction with a passerby on a city street is particularly challenging. Firstly, the passerby is likely to be subject to Attentional Overload (Milgram, 1970), a condition where excessive sensory stimulation, information and social contacts over-saturates one's attention. This inherently leads to a callous-like state in which the passersby, among other behaviours, ignore nonessential information (Inattentional Blindness) and fend-off others (Milgram, 1970 and Coon \& Mitterer, 2008). A robot attempting to instantiate an interaction with a naive passerby is likely to be faced with these challenges, the passersby are likely to ignore the robot or, upon attending to it, perceive its request for an interaction as attempted panhandling and fend-off its attempt (Goldberg, 1995 and Lankenau, 1999). However, as previously described, sociocontextual cues can individualise (in this case potentially defeating Inattentional Blindness) and communicate intention (in this case offering, which could defeat the percept of the robot's role as being that of a panhandler). Thus, it seems plausible that a robot activating the Elicit branch, through issuing specific sociocontextual cues, could establish appropriate participant roles and successfully instantiate an interaction on a 
Table 3: Participant Distribution

\begin{tabular}{|c|c|c|c|c|}
\hline & Case & Onlooker & Selected & Total \\
\hline \multirow{3}{*}{$\stackrel{\mathscr{g}}{\varrho}$} & Males & 40 & 35 & 75 \\
\hline & Females & 34 & 23 & 57 \\
\hline & Mixed & 32 & 29 & 61 \\
\hline \multirow{4}{*}{$\begin{array}{l}\bar{o} \\
\dot{\Xi} \\
\bar{U}\end{array}$} & Males & 26 & 39 & 65 \\
\hline & Females & 25 & 26 & 51 \\
\hline & Mixed & 6 & 5 & 11 \\
\hline & Total & 163 & 157 & 320 \\
\hline
\end{tabular}

city street with a task-naive passerby.

This study was conducted during business hours on a Thursday on a major city street in front of our University's main building, shown in Figure 13(a). The participants $(N=320$, the participant distribution is shown in Table 2) were typical passersby to the region, no particular demographic was evident. With regards to the table, 'Male' refers to the total number of participants in all male groups (even if it were a group of one), likewise for 'Female'. 'Mixed' refers to the total number of participants in mixed gender groups. In the instance where the Selected belonged to a 'Mixed' group, ambiguity existed upon reviewing the data as to which particular individual, and thus gender, was selected. The participants were attracted without experimenter intervention to participate in the study by the robot through its actions. The study was a $2 \times 2$ design; Gaze (Individualising \& Attention Directing) and Arm Based (Offering) Cues: Sociocontextual / Control (Non-Sociocontextual) x Participant Role: Interaction Partner (Selected) / By Stander (Onlooker). The control (non-sociocontextual) gaze and arm cues were designed with balanced movement properties to the sociocontextual cues in order to control for attention attraction through movement alone. The experimental procedure, illustrated in Figure 13(b), began with the robot randomly selecting the experimental case (Sociocontextual Cues or Control). The robot then waited until it detected a person that was heading past in the periphery (far left or right of Figure 13(b)) and selected that person as the target for interaction instantiation. The robot autonomously detected and tracked all people in the scene. The robot then: Case 1) issued initial Individualising and Offering cues at the selected person and the Attention Directing cue to the cup of pens it was holding. Reissued the Offering and Attention Directing cues upon each $\Delta d=0.5 \mathrm{~m}$ increment towards the robot taken by the participant. If the participant made no $\Delta d$ increment towards the robot then no cues other than the initial cues were issued. Or Case 2) issued the control set of cues regardless of the participants path.

Measures for this study for the selected were: their head angle relative to their direction of movement (as shown in Figure 13(b)), whether they slowed, stopped, increase proximity to the robot, gestured towards the robot (Interact) and/or approached the robot to the point where physical contact could be made with it (Approach). Measures for the onlookers were: whether they approached the robot to the point were physical contact could be made with it (Approach). The driving hypotheses for the study was that: The robot's presentation of sociocontextual cues would 


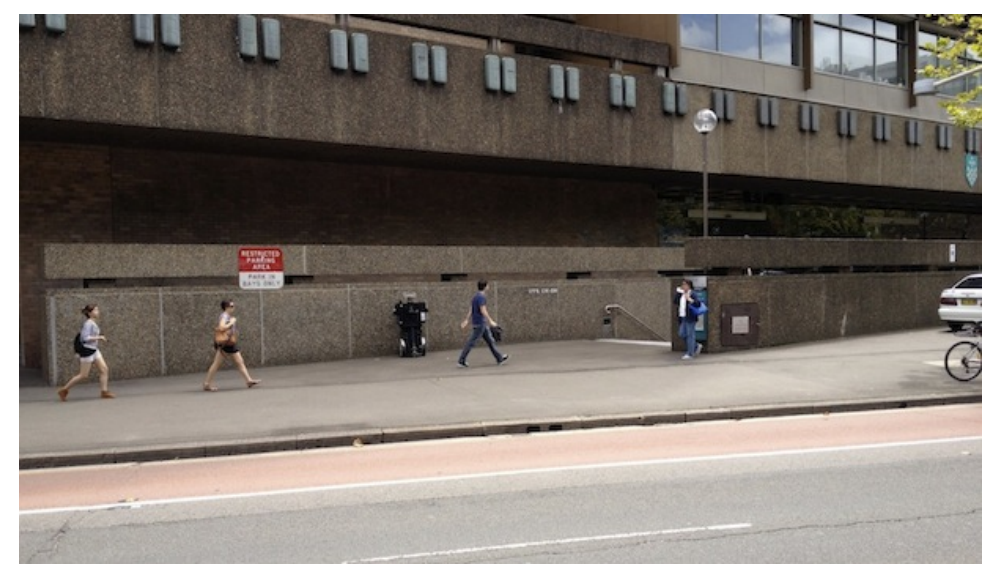

(a) The city street in front of our University

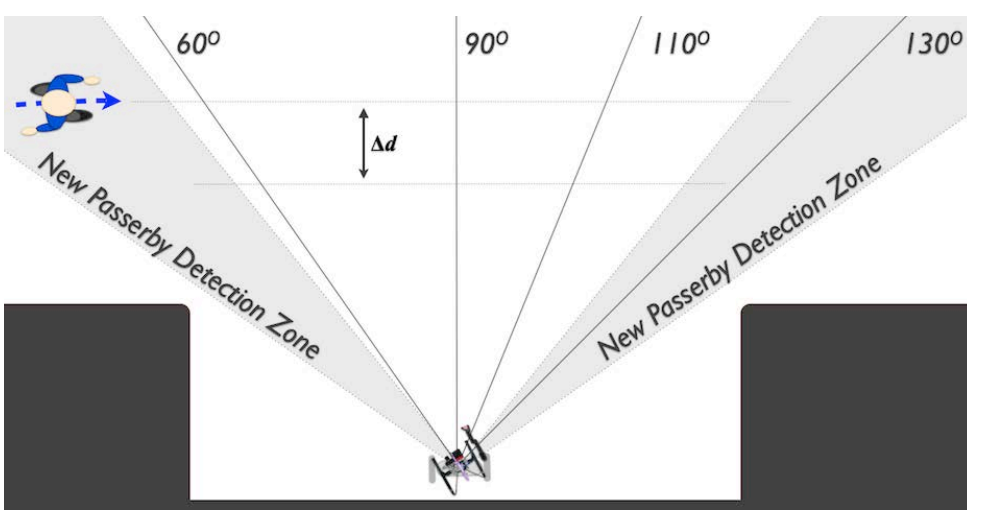

(b) An illustration of the experimental procedure

Figure 13. Experimental Setting

overcome Inattentional Blindness and the Panhandler role and allow interaction instantiation with specific passersby. Our predictions were that: upon presenting the sociocontextual cues, the robot will be more successful in instantiating an interaction with a specific passerby. Specifically, upon presenting the sociocontextual cues, 1) there would be an significantly more gaze and movement based interaction indicators, and 2) this effect will be due to the sociocontextual cues.

Tables 4(a) \& 4(b) present a selection of the key results from this study. Video from the robot's perspective was analysed post-experiment and data from participants that did not direct their heads towards the robot at any stage was excluded as it was reasonable that their behaviour was due to them having not noticed the robot. From the remaining, evidence to support our hypotheses and predictions was found. Through a series of $2 \times 2$ Fisher Exact Probability Tests and with reference to Table 4(a), it was found that significantly more passersby remain visually attending to the robot at head/movement-direction angles of $110^{\circ}$ and $130^{\circ}$ in the sociocontextual cue case, $(p=0.042$ and $p=0.040$ respectively). No significant difference was found for head/movement-direction angles of $60^{\circ}$ and $90^{\circ}$. With reference to Table 4(b), it was found that significantly more Interact and Approach indicators of an interaction were displayed by the Selected participants in the sociocontextual cues 
Table 4: Experimental Results

(a) Gaze based measures of an Interaction

\begin{tabular}{|c|c|c|c|c|c|}
\hline \multicolumn{2}{|c|}{ Looking } & $60^{\circ}$ & $90^{\circ}$ & $110^{\circ}$ & $130^{\circ}$ \\
\hline \multirow{2}{*}{$\stackrel{气}{\Xi}$} & $Y$ & 27 & 25 & 22 & 16 \\
\hline & $N$ & 26 & 28 & 30 & 34 \\
\hline \multirow{2}{*}{ 吕 } & $Y$ & 27 & 21 & 13 & 8 \\
\hline & $N$ & 28 & 34 & 40 & 44 \\
\hline
\end{tabular}

(b) Movement based measures of an interaction

\begin{tabular}{|c|c|c|c|}
\hline \multicolumn{2}{|r|}{ Case } & \multirow{2}{*}{$\begin{array}{c}\text { Interact } \\
29\end{array}$} & \multirow{2}{*}{$\begin{array}{c}\text { Approach } \\
12\end{array}$} \\
\hline 苞 & Cues & & \\
\hline$\ddot{\varpi}$ & Control & 11 & 3 \\
\hline $\begin{array}{l}\overline{\bar{v}} \\
\frac{\bar{y}}{0}\end{array}$ & Cues & 7 & 7 \\
\hline $\bar{\Xi}$ & Control & 3 & 3 \\
\hline
\end{tabular}

case than the control case, ( $p=0.002$ and $p=0.022$ respectively). It was also found that significantly more Selected participants displayed Approach indicators than did the Onlookers in the sociocontextual cues case, $(p=0.014)$. The same comparison was found to be non-significant for the control case. Finally, it was found that the participants' pass-by distance from the robot was significantly greater in the sociocontextual cue case $(\bar{x}=4.35, \sigma=1.12$ and $\bar{x}=3.86, \sigma=1.50, t(115)=1.99, p<0.05)$. Whilst this is somewhat counterintuitive, it is consistent with the findings of Mumm \& Mutlu, 2011 and has been shown to be an indication of interaction.

Firstly, it is of note that the sociocontextual cues and control cues were balanced $-52.7 \%$ and $51.6 \%$ of passersby looked at the robot once or more in the cases respectively, and this outcome distribution was found not significant through a $2 \times 2$ Fisher Exact Probability Test. This suggest that the effects seen were due to sociocontextual nature of the cues, rather than a difference in movement during the cues attracting attention, and that the sociocontextual cues were again surreptitious - the participants' behaviour was effected (as demonstrated by the results) although the participants did not display a perceived difference in visual attractiveness of the two cues cases. Furthermore, the results showed that the robot was significantly more able to complete its intended task upon presenting the sociocontextual cue. Importantly, the results from this study again demonstrate that the robot can assume the role of an Interaction Peer and overcome social tendencies, through activating the Elicit branch of our Robot Centric paradigm for HRI with sociocontextual cues, in order to increase its successfulness in achieving its task. 


\section{Concluding Discussion}

A Robot Centric paradigm for HRI which adds two branches to the traditional paradigm for HRI was presented. Empirical evidence derived from explorations of the Read and Elicit branches was presented in Sections $4 \& 5$ respectively. Through this evidence, it was demonstrated that both Branches are feasibly incorporated into HRI and moreover, both yield significant contributions to a robot's ability to successfully derive and/or complete tasks. For instance, in Section 4 it was shown that interaction-context knowledge accrued by the robot through sensing and perception could be exploited to derive informed action plans. Furthermore, in Section 5 it was shown that the robot can significantly increase its successful task completion rate by overcoming social tendencies through activating the Elicit branch of our Robot Centric paradigm for HRI with sociocontextual cues.

Together the results presented here within illustrate the need for a revision of the traditional model for HRI and provide encouraging preliminary results towards exploring our paradigm. Whilst there is still considerable space around our Robot Centric paradigm for HRI remaining open for exploration, from our aforementioned work it is our perspective that social robotics will not fulfil their full potential whilst the associated HRI confines them to the role of a Task Completer. Situations in which the humans that the robot is sharing the environment with are not privy to the robot's task/intentions are foreseeable. As demonstrated here within, in such situations the robot can increase the likelihood of successful task completion by modelling the interaction on our paradigm for Robot Centric HRI.

\section{Acknowledgements}

This work is supported by the University of Technology Sydney's (UTS) Centre for Autonomous Systems, RobotAssist (www.robotassist.org) and the University of Technology, Sydney. Further information on our activities can be found in the blog at www.nkHRI.com .

\section{References}

Alempijevic, A., Caraian, S., Egan-Wyer, D., Dissanayake, G., Fitch, R., Hengst, B., et al. (2011). RobotAssistRoboCup@Home 2011. In Technical Description Paper - RoboCup 2011 (pp. 1-8). Istanbul, Turkey.

Alempijevic, A., Dissanayake, G., Fitch, R., Hengst, B., Hordern, D., Kirchner, N., et al. (2010). Human-robot interaction research platform: CAS@Home. In Technical Description Paper - RoboCup 2010 (pp. 1-8). Singapore, Singapore.

Australia, \& Swan, W. (2010). 2010 Australia to 2050: future challenges. Treasurys 2010 Intergenerational Report (W. Swan, Ed.). Canberra: Commonwealth of Australia.

Bock, T., Linner, T., \& Ikeda, W. (2012). Exoskeleton and humanoid robotic technology in construction and built environment (D. R. Zaier, Ed.). The Future of Humanoid Robots - Research and Applications. Available from $10.5772 / 27694$

Brown, R. (1965). The explanation of behaviour. Philosophy, 40(154), pp. 344-348. Available from http://www.jstor.org/stable/3748952

Burke, J., \& Murphy, R. (2004). Human-robot interaction in USAR technical search: two heads are better than one. In Robot and human interactive communication, ROMAN 2004. 13th IEEE international workshop on (pp. 307-312). Available from 10.1109/ROMAN.2004.1374778

Caraian, S., \& Kirchner, N. (2010). Robust manipulability-centric object detection in time-of-flight camera point clouds. In Proceedings of the Australasian conference on robotics and automation 2010 (ACRA 2010) (pp. 1-9). Brisbane, Australia: Australian Robotics and Automation Association Inc. Available from http://hdl. handle.net/10453/16342

Carton, J., Kessler, E. A., \& Pape, C. L. (1999). Nonverbal decoding skills and relationship well-being in adults. Journal of Nonverbal Behavior, 23,91-100. Available from 10.1023/A:1021339410262

Colman, A. M. (2009). A dictionary of psychology (3rd ed. ed.). Oxford University Press, Oxford ; New York $\therefore$ 
Coon, D., \& Mitterer, J. O. (2008). Psychology: Modules for active learning. Wadsworth. Available from http: / /books.google.com.au/books?id=UXHHq1 jVmHAC

Cowley, S. J. (2008). Social robotics and the person problem. In Proceedings of AISB 2008 convention. Aberdeen, Scotland.

Dautenhahn, K. (2007). Socially intelligent robots: dimensions of human-robot interaction. Philosophical Transactions of the Royal Society of London - Series B: Biological Sciences, 362(1480), 679-704. Available from $10.1098 /$ rstb.2006.2004

Dautenhahn, K., \& Saunders, J. (2011). New frontiers in human-robot interaction. John Benjamins Publishing Company. Available from http://books.google.com.au/books?id=_FlP3ZBhq6oC

Diener, E., Fraser, S. C., Beaman, A. L., \& Kelem, R. T. (1976). Effects of deindividuation variables on stealing among halloween trick-or-treaters. Journal of Personality and Social Psychology, 33(2), 178183. Available from $10.1037 / 0022-3514.33 .2 .178$

Dusan, J., \& Traum, D. R. (2007). Dynamic movement and positioning of embodied agents in multiparty conversations. In Proceedings 6th international joint conference on autonomous agents and multiagent systems (AAMAS) (pp. 1-3). Honolulu, Hawaii. Available from $10.1145 / 1329125.1329142$

Finke, M., Koay, K. L., Dautenhahn, K., Nehaniv, C. L., Walters, M. L., \& Saunders, J. (2005). Hey, i'm over here - how can a robot attract people's attention. In IEEE international workshop on robot and human interactive communication (pp. 7-12). Available from 10.1109/ROMAN.2005.1513748

Geller, E., Siddiqui, N., J.M.Wu, \& Visco, A. (2008). Short-term outcomes of robotic sacrocolpopexy compared with abdominal sacrocolpopexy. Obstet Gynecol, 112(6), 1201-1206. Available from $10.1097 /$ AOG.0b013e31818ce394

Gieselmann, P., \& Waibel, A. (2005). What makes human-robot dialogues struggle? In Proceedings 9th workshop on the semantics and pragmatics of dialogue (DIALOR). Nancy, France.

Goldberg, T. (1995). Altruism towards panhandlers: Who gives? Human Nature, 6, 79-89. Available from $10.1007 / \mathrm{BF} 02734137$

Gordon, C., Churchill, T., Clauser, C., Bradtmiller, B., \& McConville, J. (1989). 1988 anthropometric survey of U.S. Army personnel: Methods and summary statistics (Tech. Rep.). United States Army Natick, Research, Development and Engineering Center.

Goren, C. C., Sarty, M., \& Wu, P. Y. K. (1975). Visual following and pattern discrimination of face-like stimuli by newborn infants. Pediatrics, 56(4), 544-549. Available from http://pediatrics. aappublications.org/content/56/4/544.abstract

Groom, V. (2008). What's the best role for a robot? cybernetic models of existing and proposed humanrobot interaction structures. In Proceedings of the international conference on informatics in control, automation, and robotics (ICINCO) 2008 (pp. 2315-2320). Funchal, Portugal.

Guerin, B. (1999). Social behaviors as determined by different arrangements of social consequences: Social loafing, social facilitation, deindividuation, and a modified social loafing. Psychological Record, 49(4), $565-578$.

Guerin, B. (2003). Social behaviors as determined by different arrangements of social consequences: Diffusion of responsibility effects with competition. The Journal of Social Psychology, 143(3), 313-329. Available from $10.1080 / 00224540309598447$

Hearst, M. (1999). Mixed-initiative interaction. IEEE Intelligent Systems, 14(5), 14-23.

Hilsenbeck, B., \& Kirchner, N. (2011). Listening for people: Exploiting the spectral structure of speech to robustly perceive the presence of people. In Intelligent robots and systems (IROS), 2011 IEEE/RSJ international conference on (pp. 2903-2909). Available from 10.1109/IROS.2011.6094825

Hordern, D., \& Kirchner, N. (2010). Robust and efficient people detection with 3-d range data using shape matching. In Proceedings of the Australasian conference on robotics and automation 2010 (ACRA 2010) (pp. 1-9). Brisbane, Australia: Australian Robotics and Automation Association Inc. Available from http: / / hdl.handle.net/10453/16431

Huang, C.-M., \& Mutlu, B. (2012). Robot behavior toolkit: generating effective social behaviors for robots. In Proceedings of the 7th annual ACM/IEEE international conference on human-robot interaction (pp. 25-32). New York, NY, USA: ACM. Available from 10.1145/2157689.2157694 
Imamoglu, E. O. (1975). Children's awareness and usage of intention cues. Child Development, 46, 39-45. Available from http: //www. jstor.org/stable/1128831

Ishiguro, H. (2011). Philosophy of android. Kagaku Tetsugaku, 44(2), 2-17.

Kendon, A. (1973). The role of visible behavior in the organization of social interaction. New York: Academic Press Ltd.

Kendon, A. (1977). Spatial organization in social encounters: The F-formation system. Lisse: DeRidder Press.

Kendon, A. (2004). Gesture: Visible action as utterance. Cambridge, UK: Cambridge University Press. Available from http: / /books.google.com.au/books?id=hDXnnzmDkOkC

Kiesler, S., Siegel, J., \& McGuire, T. W. (1984). Social psychological aspects of computermediated communication. San Francisco, CA: Morgan Kaufmann Publishers Inc. Available from http: / / dl.acm.org/citation. cfm?id=49504.49528

Kirchner, N., Alempijevic, A., \& Dissanayake, G. (2011). Nonverbal robot-group interaction using an imitated gaze cue. In Proceedings of the 6th ACM/IEEE international conference on human robot interaction (pp. 1-8). Lausanne, Switzerland. Available from 10.1145/1957656.1957824

Kirchner, N., Alempijevic, A., \& Virgona, A. (2012). Head-to-shoulder signature for person recognition. In Robotics and automation, 2012. proceedings. ICRA '12. IEEE international conference on (pp. 1-6). Available from 10.1109/ICRA.2012.6224901

Kirchner, N., \& et al. (2010). Robotassist - a platform for human robot interaction research. In Proceedings of the Australasian conference on robotics and automation 2010 (ACRA 2010) (pp. 110). Brisbane, Australia: Australian Robotics and Automation Association Inc. Available from http://hdl.handle.net/10453/16459

Knapp, M. L., \& Hall, J. A. (2009). Nonverbal communication in human interaction. Wadsworth, Cengage Learning. Available from http://books.google.com.au/books?id=xgYRAQAAIAAJ

Lankenau, S. E. (1999). Panhandling repertoires and routines for overcoming the nonperson treatment. Deviant Behavior, 20(2), 183-206. Available from $10.1080 / 016396299266551$

Mehrabian, A. (1971). Silent messages. Belmont, CA: Wadsworth. Available from http://books.google.com.au/books?id=Ast-AAAAMAAJ

Mehrabian, A. (2007). Nonverbal communication. Aldine Transaction. Available from http://books.google.no/books?id=Xt-YALu9CGwC

Milgram, S. (1970). The experience of living in cities. Science, 167, 1461-1468. Available from http://books.google.com.au/books?id=7kHaNAAACAAJ

Mumm, J., \& Mutlu, B. (2011). Human-robot proxemics: physical and psychological distancing in humanrobot interaction. In Proceedings of the 6th annual ACM/IEEE international conference on human-robot interaction (pp. 331-338). Lausanne, Switzerland. Available from 10.1145/1957656.1957786

Mutlu, B., Bartneck, C., Ham, J., Evers, V., \& Kanda, T. (2011). Social robotics: Third international conference on social robotics, ICSR 2011, amsterdam, the netherlands, november 24-25, 2011. proceedings. Springer. Available from http://books.google.com.au/books? id=5lBYuxnws.SAC

O'Callaghan, S., Singh, S., Alempijevic, A., \& Ramos, F. (2011). Learning navigational maps by observing human motion patterns. In Robotics and automation (ICRA), 2011 IEEE international conference on (pp. 4333-4340). Available from 10.1109/ICRA.2011.5980478

Postmes, T., \& Spears, R. (1998). Deindividuation and antinormative behavior: A meta-analysis. Psychological Bulletin, 123(3), 238- 259.

Poyatos, F. (1997). Nonverbal communication and translation: New perspectives and challenges in literature, interpretation and the media. J. Benjamins. Available from http://books.google.com.au/books?id=0olsAAAAIAAJ

Quan, W., Niwa, H., Ishikawa, N., Kobayashi, Y., \& Kuno, Y. (2011). Assisted-care robot based on sociological interaction analysis. Computers in Human Behavior, 27(5), 1527-1534. Available from $10.1016 / j . c h b .2010 .10 .022$

Rahimi, M., \& Karwowski, W. (1990). A research paradigm in human-robot interaction. International Journal of Industrial Ergonomics, 5(1), 59-71. Available from 10.1016/0169-8141 (90) 90028-Z

Richards, D., Paul, G., Webb, S., \& Kirchner, N. (2010). Manipulator-based grasping pose selection by 
means of task-objective optimisation. In Proceedings of the Australasian conference on robotics and automation 2010 (ACRA 2010) (pp. 1-9). Brisbane, Australia: Australian Robotics and Automation Association Inc. Available from http://hdl. handle. net/10453/16450

Robins, B., Ferrari, E., Dautenhahn, K., Kronreif, G., Prazak-Aram, B., Gelderblom, G., et al. (2010). Human-centred design methods: Developing scenarios for robot assisted play informed by user panels and field trials. International Journal of Human-Computer Studies, 68(12), 873-898. Available from $10.1016 / j . i j h c s .2010 .08 .001$

Satake, S., Kanda, T., Glas, D., Imai, M., Ishiguro, H., \& Hagita, N. (2009). How to approach humans?: strategies for social robots to initiate interaction. In Proceedings of the 4th ACM/IEEE international conference on human robot interaction (pp. 109-116). La Jolla, California, USA. Available from $10.1145 / 1514095.1514117$

Severinson-Eklundh, K., Green, A., \& Huttenrauch, H. (2003). Social and collaborative aspects of interaction with a service robot. Robotics and Autonomous Systems, 42(3-4), 223-234. Available from $10.1016 /$ S0921-8890 (02) 00377-9

Syrdal, D. S., Dautenhahn, K., Koay, K. L., \& Walters, M. L. (2009). The negative attitudes towards robots scale and reactions to robot behaviour in a live human-robot interaction study. In Proceedings new frontiers in human-robot interaction, a symposium at the AISB 2009 convention (pp. 1-8). Heriot Watt University, 8-9 April 2009, Edinburgh, Scotland.

Vinciarelli, A., Pantic, M., Bourlard, H., \& Pentland, A. (2008). Social signal processing: state-ofthe-art and future perspectives of an emerging domain. In Proceedings of the 16th ACM international conference on multimedia (pp. 1061-1070). New York, NY, USA: ACM. Available from $10.1145 / 1459359.1459573$

Watzlawick, P., Bavelas, J., \& Jackson, D. (1967). Pragmatics of human communication: A study of interactional patterns, pathologies, and paradoxes. Norton. Available from http://books.google.com.au/books?id=LNuNQgAACAAJ

Authors' names and contact information: Nathan Kirchner, Centre for Autonomous Systems, Faculty of Engineering and Information Technology, University of Technology, Sydney, Sydney, Australia. Email: nathan.kirchner@uts.edu.au. Web: www.nkHRI.com. Alen Alempijevic, Centre for Autonomous Systems, Faculty of Engineering and Information Technology, University of Technology, Sydney, Sydney, Australia. Email: alen.alempijevic@uts.edu.au 